\title{
AtomSegNet and TomoFillNet - Two Deep Learning Open-Source Apps for Superresolution Processing of Atomic Resolution Images and Missing-wedge Information Inpainting in Electron Tomograms
}

\author{
Huolin Xin ${ }^{1}$, Ruoqian Lin $^{2}$, Rui Zhang ${ }^{1}$, Chunyang Wang ${ }^{1}$, Guanglei Ding ${ }^{1}$ and He Wei ${ }^{1}$ \\ ${ }^{1}$ University of California-Irvine, Irvine, California, United States, ${ }^{2}$ Brookhaven National Laboratory, \\ Upton, New York, United States
}

In this talk, we report the recent progress in the development of deep learning-based Apps in the DeepEM Laboratory of University of California, Irvine. In the past year, we have primarily focused our effort on developing two main platforms, AtomSegNet and TomoFillNet.

For the AtomSegNet, we developed a deep-learning based method for atoms segmentation and denoising of experimental atomic resolution annular dark-field scanning transmission electron microscopy (ADFSTEM) images with high robustness and precision. To tackle this problem, we created a forward model that can simulate atomic resolution ADF-STEM with synthetic scan and Poisson noises. This allows us to automatically generate experimental-like atomic-resolution STEM image datasets with known groundtruth labels for training. The established STEM images library encompassing atomic-resolution ADFSTEM images of eight different materials along multiple major zone axes. Random linear and nonlinear background interferences are added to the synthetic images to improve the robustness of our model. To further reduce the false positive rate, we also included images of small nanoparticles with tapered and sharp facet terminations. Based on our well labeled training library, we demonstrate that our encoderdecoder architectures model achieves excellent performance in atom segmentation, denoising and superresolution processing of experimental ADF-STEM images of crystal structures that were not included in the training library.

TomoFillNet sovles the missing-wedge and few-projection problems in electron tomography. Electron tomography experiment is a very dose intensive process particularly when high resolution is needed. Therefore, for materials application, there is a strong need to reduce the dosage, i.e. to reduce the number of projections needed while still maintaining the high resolution. Like all tomography techniques, when there are insufficient projections, the inverse problem of reconstructing tomograms is mathematically illposed because the solution is non-unique. In this talk, we will discuss three recently developed deep learning methods that can solve this conundrum. For the first time, by combining generative adversarial models with state-of-the-art network architectures, we show it is possible to recover the lost information and remove artifacts in the reconstructed tomograms by only acquiring data from -50 to +50 degrees $(44 \%$ reduction of dosage compared to -90 to +90 degrees full tilt series). In contrast to the conventional methods, the deep learning model shows outstanding performance for both macroscopic objects and atomistic features. This work has profound implications. It, for the first time, reveals that a single network operating in the tomogram domain is sufficient to identify priors/regularizations extracted from the training sets to inpaint the missing information. Our work offers a new and experimentally validated framework for solving the inpainting problems in other tomographic and 3D imaging techniques.

\section{References}

[1] Ding, G., Liu, Y., Zhang, R. \& Xin, H. L. A joint deep learning model to recover information and reduce artifacts in missing-wedge sinograms for electron tomography and beyond. Scientific reports 9 , 12803, doi:10.1038/s41598-019-49267-x (2019). 